\title{
Hemorrhage during pregnancy in the latency interval after stereotactic radiosurgery for arteriovenous malformations
}

\author{
Clinical article
}

\author{
Daniel Tonetti, M.D., M.S., ${ }^{1}$ Hideyuki Kano, M.D., Ph.D., ${ }^{1,3}$ \\ Gregory Bowden, M.D., M.Sc., ${ }^{1,3}$ John C. Flickinger, M.D., ${ }^{2,3}$ \\ AND L. DAde LunSFORD, M.D. ${ }^{1,3}$
}

Departments of ${ }^{1}$ Neurological Surgery and ${ }^{2}$ Radiation Oncology and ${ }^{3}$ Center for Image-Guided Neurosurgery, University of Pittsburgh Medical Center, Pittsburgh, Pennsylvania

\begin{abstract}
Object. The presentation for patients with arteriovenous malformations (AVMs) is often intracranial hemorrhage; for women, this frequently occurs during the prime childbearing years. Although previous studies have addressed the risk for AVM hemorrhage during pregnancy, such studies have not assessed the risk for hemorrhage among women who become pregnant during the latency interval between stereotactic radiosurgery (SRS) and documented obliteration of the lesion. The authors sought to evaluate the risk for hemorrhage in patients who become pregnant during the latency interval after SRS.

Methods. This single-institution retrospective analysis reviewed the authors' experience with Gamma Knife SRS during 1987-2012. During this time, 253 women of childbearing age (median age 30 years, range 15-40 years) underwent SRS for intracranial AVM. The median target volume was $3.9 \mathrm{~cm}^{3}$ (range $0.1-27.1 \mathrm{~cm}^{3}$ ), and the median marginal dose was 20 Gy (range 14-38 Gy). For all patients, the date of AVM obliteration was recorded and the latency interval was calculated. Information about subsequent pregnancies and/or bleeding events during the latency interval was retrieved from the medical records and supplemented by telephone contact.

Results. AVM obliteration was confirmed by MRI or angiography at a median follow-up time of 39.3 months (range 10-174 months). There were 828.7 patient-years of follow-up within the latency interval between SRS and the date of confirmed AVM obliteration. Among nonpregnant women, 20 hemorrhages occurred before AVM obliteration, yielding an annual hemorrhage rate of $2.5 \%$ for nonpregnant women during the latency interval. Among women who became pregnant during the latency interval, 2 hemorrhages occurred over the course of 18 pregnancies, yielding an annual hemorrhage rate of $11.1 \%$ for women who become pregnant during the latency interval. For the 2 pregnant patients who experienced hemorrhage, the bleeding occurred during the first trimester of pregnancy.

Conclusions. The authors present the first series of data for women with intracranial AVMs who became pregnant during the latency interval after SRS. Hemorrhage during the latency interval occurred at an annual rate of $2.5 \%$ for nonpregnant women and $11.1 \%$ for pregnant women. The data suggest that pregnancy might be a risk factor for AVM hemorrhage during the interval between SRS and AVM obliteration. However, this suggestion is not statistically significant because only 18 patients in the study population became pregnant during the latency interval. To mitigate any increased risk for hemorrhage, patients should consider deferring pregnancy until treatment conclusion and AVM obliteration.
\end{abstract}

(http://thejns.org/doi/abs/10.3171/2014.7.GKS141297)

\section{KEY WORDS - arteriovenous malformation - stereotactic radiosurgery • Gamma Knife • pregnancy • vascular disorders}

A RTERIOVENOUS malformations (AVMs) shunt blood from the arterial system to the venous system and bypass the normal capillary bed of the brain. Although AVMs constitute only $11 \%$ of cerebrovascular brain disorders, patients with AVMs are often symptomatic and experience brain hemorrhage, headaches, or seizures, depending on AVM location.

\footnotetext{
Abbreviations used in this paper: AVM = arteriovenous malformation; GK = Gamma Knife; SRS = stereotactic radiosurgery.
}

Stereotactic radiosurgery (SRS) has proven to be an effective management strategy that significantly increases the therapeutic options for patients with AVMs. Publications indicate that AVM obliteration rates after a single procedure range from $70 \%$ to $80 \%$ over 5 years in selected groups, depending on AVM volume, dose delivered, location, and patient age. $1,5,6,8,13,19,25,29,33,35$ The median time until MRI documentation of total obliteration of AVMs after Gamma Knife SRS (Elekta AB) has been shown to be 36 months. ${ }^{16}$ 
Although various publications have addressed the risk for AVM hemorrhage during pregnancy, $4,7,11,15,28,32$ such studies have not assessed the risk for hemorrhage in women who become pregnant during the typical 3-year latency interval between SRS and documented AVM obliteration. Our objective was to evaluate the risk for hemorrhage in patients who become pregnant during the latency interval. We compared the incidence of hemorrhage among these patients with the incidence of hemorrhage among women who did not become pregnant during this interval.

\section{Methods}

We performed a retrospective review of all patients who had a confirmed angiographic diagnosis of intracranial AVM and who underwent Gamma Knife (GK) SRS at the University of Pittsburgh during 1987-2012. The University of Pittsburgh Institutional Review Board approved the study. Of the 1117 patients who met these criteria, 529 were female. To include only those patients in the typical childbearing years, we excluded female patients who were older than 40 and younger than 15 years at the time of the procedure, which left 253 patients who met the age and sex selection criteria. The study was performed by physicians who were not involved in the original radiosurgical procedure.

For all patients, the date of AVM obliteration was recorded. Information about subsequent pregnancies and/ or bleeding events that occurred during the latency interval after SRS was retrieved from the medical records and supplemented by telephone contact with patients, family members, and current treating physicians. A total of 17 (6.5\%) patients became pregnant before documentation of AVM obliteration. One patient became pregnant twice. For all 253 patients, we compiled a complete hemorrhage record and obstetrical history, when pertinent.

Stereotactic radiosurgery was performed by using one of various models (U, B, C, 4C, or Perfexion) of the Leksell Gamma Knife (Elekta AB) during 1987-2012. All patients underwent AVM SRS based on intraoperative angiography and axial plane imaging performed by using either CT or MRI (MRI after 1991). The median target volume was $3.9 \mathrm{~cm}^{3}$ (range $0.1-27.1 \mathrm{~cm}^{3}$ ), and the median margin dose used in a single procedure was 20 Gy (range 14-38 Gy). Our procedure has been outlined in detail in prior publications. ${ }^{22,23,31}$

After SRS, patients typically underwent repeat MRI or CT at 6-, 12-, 24-, and 36-month intervals. If these imaging studies suggested a high probability of AVM obliteration, patients were asked to undergo verification angiography. For each patient, we calculated the time from SRS until obliteration was documented by imaging. During this interval we evaluated bleeding events and pregnancies. The date of AVM obliteration was recorded as the date that imaging (usually MRI supplemented by angiography) confirmed an absence of AVM filling. For patients for whom total obliteration was demonstrated with both digital subtraction angiography and MRI, the date of angiography was used.

For patients who did not undergo sufficient follow- up imaging to confirm obliteration, we arbitrarily assumed the date of obliteration to be 3 years from the date of the procedure, consistent with the median time until MRI documentation of total obliteration established by prior publications. ${ }^{16,18}$ For patients whose images revealed a persistent AVM at 3 years after SRS, the most recent date of follow-up imaging that confirmed obliteration was used as the date of obliteration. Follow-up imaging was interpreted by 2 experienced neurosurgeons during reviews performed at our multidisciplinary vascular malformation conference.

Patient-years of follow-up were tallied from the date of SRS until the date of obliteration. Each pregnancy was assigned 1.0 patient-years, which consisted of 40 weeks of gestation, beginning from the last menstrual period, plus an additional 12-week postpartum interval to account for physiological changes associated with pregnancy that do not resolve immediately at the time of delivery. A casematch control analysis was then performed (1 pregnant patient to 3 nonpregnant patients [controls]) to determine statistical significance. Patients who became pregnant during the latency interval were matched to control patients by age, prior hemorrhage history, AVM location (deep vs superficial), target volume, margin dose, Spetzler-Martin grade, Pollock-Flickinger score, and follow-up duration. After case matching, the Mann-Whitney U-test for continuous data and the Fisher exact test for categorical data were used to compare both groups. All variables for case matching did not statistically differ between the groups.

\section{Results}

For 110 (43\%) of the 253 patients, AVM diagnosis was made after an initial cerebral hemorrhage. For the other patients, the AVM was diagnosed during the evaluation of symptoms that included headaches (28\%) and seizures (21\%), or it was found incidentally during the evaluation of recent mild cranial trauma (5\%). Among the 110 patients with initial intracerebral hemorrhage, 6 were pregnant at the time of the first hemorrhage. Two experienced neurosurgeons determined the Spetzler-Martin AVM grade before SRS. ${ }^{34}$ Grade I AVM was diagnosed in $15(6 \%)$ patients, Grade II in 68 (27\%), Grade III in 95 (38\%), Grade IV in 41 (16\%), and inoperable Grade VI in 34 (13\%).

\section{Hemorrhage Frequency}

Annual rates of hemorrhage were calculated on the basis of years of follow-up and total number of hemorrhages; these rates account for the total number of hemorrhages experienced by patients during the latency interval. A total of 828.7 patient-years of follow-up occurred during the latency interval between SRS and the date of confirmed AVM obliteration (Table 1). This number accounts for 810.7 patient-years of follow-up during which patients were not pregnant and 18.0 years of follow-up during which patients were pregnant. Median followup time for all patients was 36 months (range 10-174 months). During the latency interval, 20 hemorrhages occurred in nonpregnant patients, equating to an annual hemorrhage rate of $2.5 \%(20 / 810.7)$. Of these 20 patients, $12(60 \%)$ had previously experienced AVM hemorrhage. 
TABLE 1: Characteristics of the patient population

\begin{tabular}{lc}
\hline \multicolumn{1}{c}{ Variable } & Value \\
\hline no. of patients & 253 \\
mean age at SRS, yrs (range) & $29.6(15-40)$ \\
mean SRS latency interval, yrs \pm SD & $3.2 \pm 1.9$ \\
total patient-years of follow-up & 828.7 \\
no. of pregnancies & 18 \\
no. of hemorrhages during pregnancy & 2 \\
no. of hemorrhages in patients not pregnant at & 20 \\
$\quad$ the time & \\
\hline
\end{tabular}

The 110 patients with intracranial hemorrhage at initial presentation accounted for 327.2 patient-years of follow-up. Of these 110 patients, $13(12 \%)$ sustained repeated intracerebral hemorrhages during the latency interval, yielding an annual rebleed rate of $4.0 \%$ (13/327.2) until obliteration. Of the 13 rebleeding events, 4 were fatal.

During the latency period, 18 pregnancies occurred among 17 patients. Two hemorrhage events occurred within the latency period, resulting in a calculated annual hemorrhage rate during pregnancy of $11.1 \%(2 / 18)$ (Table 2 ). For each of these 2 patients, hemorrhages occurred during the first trimester of pregnancy. The case-match control analysis did not reveal a statistically significant association between pregnancy and nonpregnancy rates of hemorrhage $(\mathrm{p}=0.696)$.

\section{Illustrative Cases}

\section{Case 1}

A 19-year-old woman with right-sided weakness and blurry vision at initial presentation underwent GK SRS for an $18-\mathrm{cm}^{3}$ Spetzler-Martin Grade VI left thalamic AVM (Fig. 1) that had multiple arterial feeders and draining veins, deep venous drainage, and a varix but no prenidal or intranidal aneurysms. The SRS was performed in 2 stages separated by a 3-month interval. The minimal margin dose to the entire AVM was $16 \mathrm{~Gy}$. One month after undergoing SRS, the patient became pregnant. At Week 5 of gestation, she experienced a severe headache and new dense right hemiparesis caused by AVM rupture. While under observation, the patient sustained a second hemorrhage at Week 10 of gestation, resulting in coma. Placement of an external ventricular drain was followed by slow recovery of consciousness. The patient experienced spontaneous abortion during Week 12 of gestation. At the 2-year follow-up visit, the patient was awake and alert but with residual right hemiparesis. Follow-up imag-

TABLE 2: Annual rates of hemorrhage during SRS latency period

\begin{tabular}{lc}
\hline \multicolumn{1}{c}{ Variable } & Rate (\%) \\
\hline hemorrhage & 2.7 \\
rebleed & 4.0 \\
hemorrhage when not pregnant & 2.5 \\
hemorrhage when pregnant & 11.1 \\
\hline
\end{tabular}

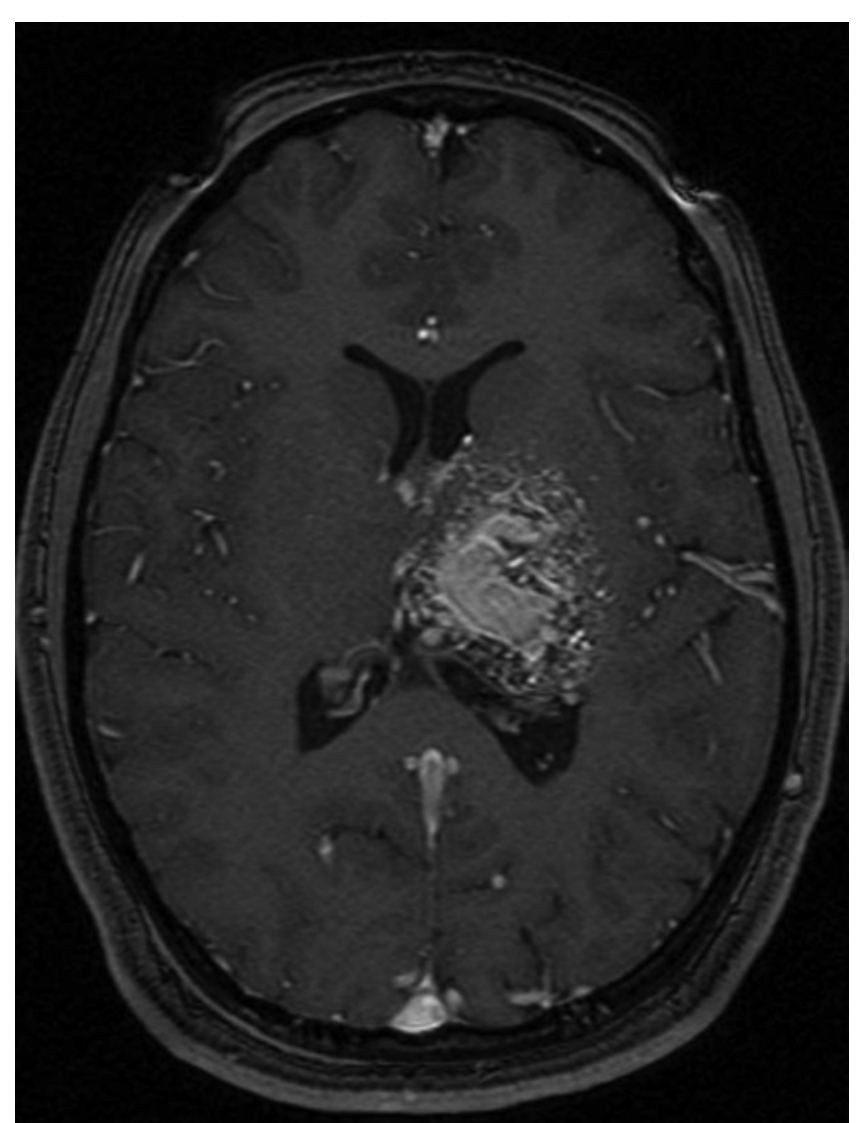

Fig. 1. Case 1. MR image of a 19-year-old woman with weakness and blurry vision secondary to a Grade VI left thalamic AVM.

ing at 30 months after the first staged procedure demonstrated a reduction in AVM size, and the patient recently underwent repeat GK SRS with a minimal margin dose of $18 \mathrm{~Gy}$ to the entire residual AVM.

Case 2

A 32-year-old woman with a 1-year history of headaches was found to have a right medial anterior temporal Spetzler-Martin Grade III (S2V1E0) AVM (Fig. 2), which had several arterial feeders and draining veins, deep venous drainage, and a varix but no prenidal or intranidal aneurysms. SRS was performed for this $10-\mathrm{cm}^{3}$ AVM; 17 Gy was delivered at the margin in a single SRS session. Fourteen months later, the patient sustained an AVM hemorrhage and was subsequently found to be in her 6th week of pregnancy. She underwent craniotomy for clot evacuation and partial AVM removal. Four weeks later, at Week 10 of gestation, the patient experienced a spontaneous abortion. Three years after SRS and subsequent clot evacuation, the patient was neurologically intact and angiography confirmed AVM obliteration.

\section{Discussion}

\section{AVM Hemorrhage Risk Among Women}

Among women, AVMs are most likely to hemorrhage during the prime childbearing years; one large study of 


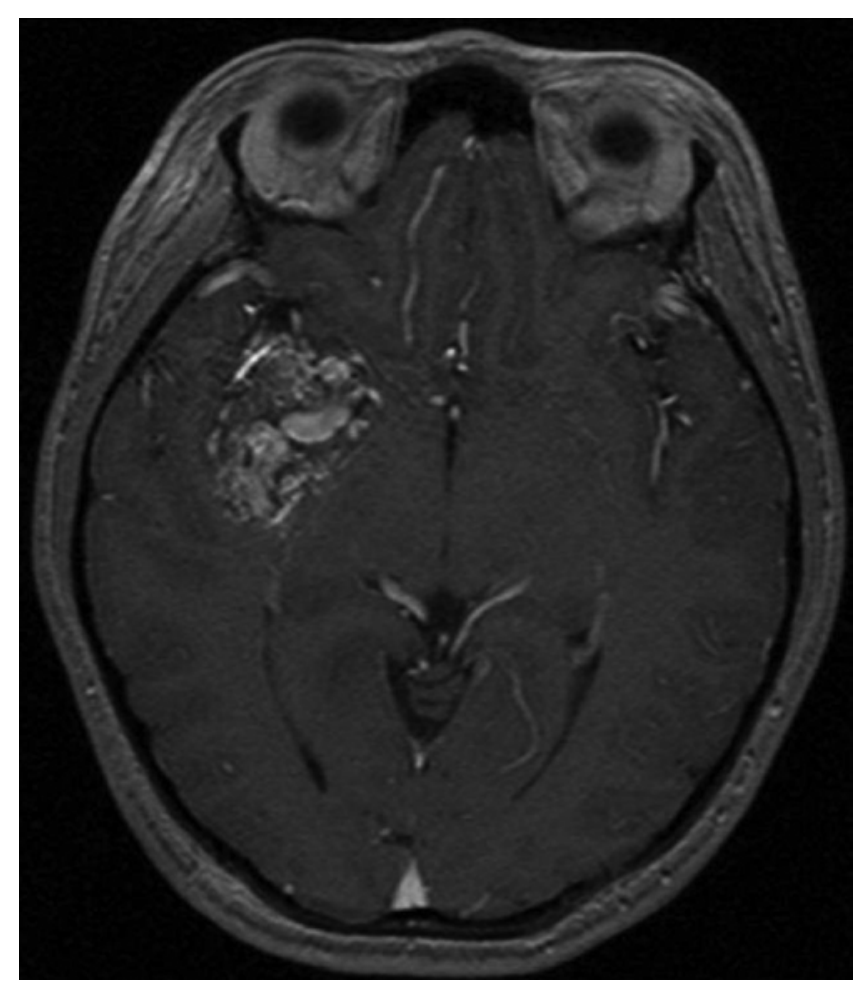

FIG. 2. Case 2. MR image of a 32-year-old woman with a 1-year history of headaches secondary to a right medial anterior temporal Grade III AVM.

women reported a mean $( \pm \mathrm{SD})$ age at AVM diagnosis of $27.5 \pm 12.9$ years. ${ }^{15}$ Additionally, most AVMs discovered during pregnancy are discovered as a result of cerebral hemorrhage. ${ }^{15,32}$ Taken together, these findings seem to indicate that among women, the likelihood of hemorrhage is higher during childbearing years and pregnancy might increase the risk for hemorrhage.

The most important outcome variable for patients who undergo treatment for intracranial AVM is reduction or elimination of future risk for hemorrhage. Studies of the natural history of AVMs have reported annual hemorrhage rates of $1.7 \%-2.7 \%$ for previously unruptured AVMs and 3.7\%-5.5\% for AVMs that have previously bled. ${ }^{12}$ The risk for rebleeding is highest during the 1st year after an initial bleed; rates range from $6 \%$ to $15 \%$. 2,9,10,12,27,30 The cumulative rate for AVM hemorrhage among patients who have undergone GK SRS has been reported to be $7.5 \%$ at 10 years. ${ }^{16}$ After the AVM has been radiographically obliterated, the cumulative lifetime risk for an AVM hemorrhage is $1 \% .^{26}$

Studies suggest that the strongest risk factors for AVM hemorrhage are deep venous drainage, large volume, prior hemorrhage, and presence of associated aneurysms. ${ }^{3,14,17}$ Although pregnancy is generally considered to be an additional risk factor for hemorrhage, confirming data are relatively scarce. Gross and $\mathrm{Du}^{11}$ recently reported that annual hemorrhage rates among 54 women with AVMs were $10.8 \%$ in pregnant women and $1.1 \%$ in nonpregnant women $(\mathrm{p}=0.000223)$. Such a large increase in risk for AVM hemorrhage suggests another reason why women with AVMs discovered during their childbearing years should consider AVM treatment. In contrast, Horton et al. retrospectively analyzed this risk among 451 women (540 pregnancies) with untreated AVMs who eventually underwent radiosurgery. They demonstrated a $3.5 \%$ hemorrhage rate during the year of pregnancy versus an annual hemorrhage rate of $3.1 \%$ outside the year of pregnancy $(\mathrm{p}=0.35) .{ }^{15}$ These authors concluded that pregnancy was not a risk factor for hemorrhage in women who had not previously experienced hemorrhage. A separate study of women undergoing radiosurgery, which used the same outcome analysis reported by Horton et al., identified a $9.3 \%$ annual rate of hemorrhage for women during pregnancy and a $4.5 \%$ annual rate for women older than the typical childbearing age.

In a case-crossover study of a large series of 393 patients with ruptured AVMs, ${ }^{21}$ Liu et al. recently reported no increased risk for AVM rupture during pregnancy (OR $0.71,95 \%$ CI $0.61-0.82)$. Their study and ours differ in numerous ways that contribute to the differing conclusions. In the Liu et al. study, all patients analyzed had experienced AVM hemorrhage, and patients who bled were less likely to get pregnant again. Because they did not follow up the patients who experienced hemorrhage before pregnancy to determine whether a subsequent hemorrhage occurred during pregnancy, these authors eliminated from analysis the subgroup of women who become pregnant after a first AVM hemorrhage. Furthermore, in the same study, the length of pregnancy for the 452 reported pregnancies (in the 393 patients) is unknown for most patients; of the 12 study patients who experienced hemorrhage during pregnancy, none carried the child beyond 38 weeks. Of the 440 other pregnancies, the number of women who carried the child to full term is unknown. Although this study is a markedly large one determining that pregnancy is not a risk factor for AVM hemorrhage, calculating hemorrhage rates during pregnancy on the basis of the assumption that all pregnancies completed the full term, in addition to eliminating the subgroup of women who become pregnant after hemorrhage (thereby eliminating prior hemorrhage as a risk factor for their pregnancy analysis), both artificially lowers the hemorrhage rate and may explain why their results differ from ours.

Although not statistically significant, our data demonstrate an elevated risk for hemorrhage during pregnancy in the latency interval after SRS. The lack of statistical significance can be largely attributed to small sample size; of the 253 patients studied, only 18 patients became pregnant during the latency interval. Many women contacted for this study stated that they had been counseled at the time of AVM diagnosis that pregnancy could increase their risk for hemorrhage, which probably contributed to the relatively low rate of pregnancy in our study population.

\section{Vascular Changes During Pregnancy}

Previous studies have demonstrated histologic changes in arteries and arterioles of pregnant women, thought to be mediated by progesterone; the changes included fragmentation of reticular fibers and loss of normal corrugation of elastic fibers, which might play a role in the increased risk for AVM hemorrhage during pregnancy. ${ }^{24}$ 
This finding suggests that the risk for hemorrhage during pregnancy would peak in the third trimester because circulating levels of progesterone increase throughout pregnancy; however, our data do not support this concept.

Prior studies have demonstrated variation with respect to the timing of hemorrhage during pregnancy. ${ }^{11,15,20}$ Of 17 total hemorrhages in the study by Horton et al., ${ }^{15} 4$ $(23.5 \%)$ occurred during the first trimester, $6(35.3 \%)$ during the second trimester, 5 (29.4\%) during the third trimester, and $2(11.8 \%)$ during the puerperium. This finding suggests a relatively consistent risk for hemorrhage throughout pregnancy but has been challenged by other studies. In a recent study by Gross and Du, of 5 hemorrhages, ${ }^{11} 4(80 \%)$ occurred during the third trimester. In contrast, in our study population, both hemorrhages that occurred in pregnant women during the GK surgery latency interval occurred during the first trimester of pregnancy. These data for an admittedly small number of patients suggest that no definite correlation is yet known to exist between gestational age and risk for intracranial hemorrhage from AVM.

\section{Management of Patients Who Become Pregnant During the SRS Latency Interval}

Patients who become pregnant during the latency interval present additional management challenges. The decision whether to offer the patient additional treatment measures is a difficult one that often requires consultation with clinicians from obstetric, anesthesiology, and neurosurgical services. This decision is based on risks to the patient inherent to additional treatment as well as the potential risk to the fetus during the intervention and associated diagnostic testing. At our institution, we favor conservative management for this unique subset of patients and do not recommend that patients who become pregnant after SRS undergo subsequent embolization or microsurgery, regardless of gestational age. Throughout the patient's pregnancy, careful consideration should be paid to the management of blood pressure because hypertension increases the risk for hemorrhage and hypotension can compromise uterine blood flow and risk fetal distress. During delivery, anesthetic management should be predicated on minimizing cardiovascular changes. The available data suggest that risk for hemorrhage is no higher with vaginal delivery than with delivery by cesarean section; ${ }^{15}$ however, with an abundance of caution, we recommend that all AVM patients who become pregnant undergo cesarean section delivery.

\section{Conclusions}

Intracranial AVMs are congenital malformations that can rupture and cause intracerebral hemorrhage at any time throughout life, leading to potentially devastating outcomes. Many women of childbearing age with AVMs, faced with the danger of potentially increasing their risk for hemorrhage, decide not to become pregnant. However, some women do consider the possibility of pregnancy and may benefit from a quantitative assessment of the risk for hemorrhage during pregnancy in the interval after GK SRS. We present the first series of data for women with intracranial AVMs who became pregnant during the latency interval after SRS. Although not statistically significant, our data suggest that pregnancy is a risk factor for hemorrhaging between radiosurgery and AVM obliteration.

Given these findings it is our recommendation that when AVMs are discovered in nonpregnant women of childbearing age, these patients should be counseled regarding the likely elevated risk for hemorrhage should they become pregnant. To mitigate this risk, these patients should strongly consider deferring pregnancy until treatment is concluded and the AVM is obliterated.

\section{Acknowledgment}

We thank Professor Douglas Kondziolka, M.D. (New York University Langone Medical Center), for significant contributions to patient management.

\section{Disclosure}

Dr. Lunsford is a consultant for and stockholder of Elekta AB. The work described in this report was funded by the Center for Image-Guided Neurosurgery, Pittsburgh, Pennsylvania.

Author contributions to the study and manuscript preparation include the following. Conception and design: Kano, Tonetti, Bowden, Lunsford. Acquisition of data: Kano, Tonetti, Bowden. Analysis and interpretation of data: Kano, Tonetti, Bowden. Drafting the article: Tonetti, Bowden. Critically revising the article: Kano, Tonetti, Bowden, Lunsford. Reviewed submitted version of manuscriptt: Kano, Tonetti, Bowden, Lunsford. Approved the final version of the manuscript on behalf of all authors: Kano. Statistical analysis: Kano, Tonetti, Bowden. Administrative/technical/material support: all authors. Study supervision: all authors.

\section{References}

1. Colombo F, Benedetti A, Pozza F, Marchetti C, Chierego G: Linear accelerator radiosurgery of cerebral arteriovenous malformations. Neurosurgery 24:833-840, 1989

2. Crawford PM, West CR, Chadwick DW, Shaw MD: Arteriovenous malformations of the brain: natural history in unoperated patients. J Neurol Neurosurg Psychiatry 49:1-10, 1986

3. da Costa L, Wallace MC, Ter Brugge KG, O'Kelly C, Willinsky RA, Tymianski M: The natural history and predictive features of hemorrhage from brain arteriovenous malformations. Stroke 40:100-105, 2009

4. Dias MS, Sekhar LN: Intracranial hemorrhage from aneurysms and arteriovenous malformations during pregnancy and the puerperium. Neurosurgery 27:855-866, 1990

5. Fleetwood IG, Steinberg GK: Arteriovenous malformations. Lancet 359:863-873, 2002

6. Flickinger JC, Kondziolka D, Maitz AH, Lunsford LD: An analysis of the dose-response for arteriovenous malformation radiosurgery and other factors affecting obliteration. Radiother Oncol 63:347-354, 2002

7. Forster DM, Kunkler IH, Hartland P: Risk of cerebral bleeding from arteriovenous malformations in pregnancy: the Sheffield experience. Stereotact Funct Neurosurg 61 (Suppl 1):20-22, 1993

8. Friedman WA, Bova FJ: Linear accelerator radiosurgery for arteriovenous malformations. J Neurosurg 77:832-841, 1992

9. Fults D, Kelly DL Jr: Natural history of arteriovenous malformations of the brain: a clinical study. Neurosurgery 15:658662, 1984

10. Graf CJ, Perret GE, Torner JC: Bleeding from cerebral arteriovenous malformations as part of their natural history. J Neurosurg 58:331-337, 1983 
11. Gross BA, Du R: Hemorrhage from arteriovenous malformations during pregnancy. Neurosurgery 71:349-356, 2012

12. Gross BA, Du R: Natural history of cerebral arteriovenous malformations: a meta-analysis. Clinical article. J Neurosurg 118:437-443, 2013

13. Herbert C, Moiseenko V, McKenzie M, Redekop G, Hsu F, Gete E, et al: Factors predictive of obliteration after arteriovenous malformation radiosurgery. Can J Neurol Sci 38:845850,2011

14. Hernesniemi JA, Dashti R, Juvela $S$, Väärt $K$, Niemelä $M$, Laakso A: Natural history of brain arteriovenous malformations: a long-term follow-up study of risk of hemorrhage in 238 patients. Neurosurgery 63:823-831, 2008

15. Horton JC, Chambers WA, Lyons SL, Adams RD, Kjellberg RN: Pregnancy and the risk of hemorrhage from cerebral arteriovenous malformations. Neurosurgery 27:867-872, 1990

16. Kano H, Kondziolka D, Flickinger JC, Park KJ, Iyer A, Yang $\mathrm{HC}$, et al: Stereotactic radiosurgery for arteriovenous malformations after embolization: a case-control study. Clinical article. J Neurosurg 117:265-275, 2012

17. Kano H, Kondziolka D, Flickinger JC, Yang HC, Park KJ, Flannery TJ, et al: Aneurysms increase the risk of rebleeding after stereotactic radiosurgery for hemorrhagic arteriovenous malformations. Stroke 43:2586-2591, 2012

18. Kano H, Lunsford LD, Flickinger JC, Yang HC, Flannery TJ, Awan NR, et al: Stereotactic radiosurgery for arteriovenous malformations, Part 1: management of Spetzler-Martin Grade I and II arteriovenous malformations. Clinical article. J Neurosurg 116:11-20, 2012

19. Karlsson B, Kihlström L, Lindquist C, Steiner L: Gamma knife surgery for previously irradiated arteriovenous malformations. Neurosurgery 42:1-6, 1998

20. Kelly DL Jr, Alexander E Jr, Davis CH Jr, Maynard DC: Intracranial arteriovenous malformations: clinical review and evaluation of brain scans. J Neurosurg 31:422-428, 1969

21. Liu XJ, Wang S, Zhao YL, Teo M, Guo P, Zhang D, et al: Risk of cerebral arteriovenous malformation rupture during pregnancy and puerperium. Neurology 82:1798-1803, 2014

22. Lunsford LD, Kondziolka D, Flickinger JC, Bissonette DJ, Jungreis CA, Maitz AH, et al: Stereotactic radiosurgery for arteriovenous malformations of the brain. J Neurosurg 75:512-524, 1991

23. Maesawa S, Flickinger JC, Kondziolka D, Lunsford LD: Repeated radiosurgery for incompletely obliterated arteriovenous malformations. J Neurosurg 92:961-970, 2000

24. Manalo-Estrella P, Barker AE: Histopathologic findings in human aortic media associated with pregnancy. Arch Pathol 83:336-341, 1967

25. Maruyama K, Kawahara N, Shin M, Tago M, Kishimoto J, Kurita $\mathrm{H}$, et al: The risk of hemorrhage after radiosurgery for cerebral arteriovenous malformations. N Engl J Med 352:146-153, 2005

26. Maruyama K, Shin M, Tago M, Kishimoto J, Morita A, Kawahara N: Radiosurgery to reduce the risk of first hemorrhage from brain arteriovenous malformations. Neurosurgery 60: 453-459, 2007

27. Mast H, Young WL, Koennecke HC, Sciacca RR, Osipov A, Pile-Spellman J, et al: Risk of spontaneous haemorrhage after diagnosis of cerebral arteriovenous malformation. Lancet 350:1065-1068, 1997

28. Michelsen WJ: Natural history and pathophysiology of arteriovenous malformations. Clin Neurosurg 26:307-313, 1979

29. Ogilvy CS, Stieg PE, Awad I, Brown RD Jr, Kondziolka D, Rosenwasser R, et al: AHA Scientific Statement: Recommendations for the management of intracranial arteriovenous malformations: a statement for healthcare professionals from a special writing group of the Stroke Council, American Stroke Association. Stroke 32:1458-1471, 2001

30. Ondra SL, Troupp H, George ED, Schwab K: The natural history of symptomatic arteriovenous malformations of the brain: a 24-year follow-up assessment. J Neurosurg 73:387391, 1990

31. Pollock BE, Flickinger JC, Lunsford LD, Maitz A, Kondziolka D: Factors associated with successful arteriovenous malformation radiosurgery. Neurosurgery 42:1239-1247, 1998

32. Robinson JL, Hall CS, Sedzimir CB: Arteriovenous malformations, aneurysms, and pregnancy. J Neurosurg 41:63-70, 1974

33. Skjøth-Rasmussen J, Roed H, Ohlhues L, Jespersen B, Juhler M: Complications following linear accelerator based stereotactic radiation for cerebral arteriovenous malformations. Int J Radiat Oncol Biol Phys 77:542-547, 2010

34. Spetzler RF, Martin NA: A proposed grading system for arteriovenous malformations. J Neurosurg 65:476-483, 1986

35. Yamamoto Y, Coffey RJ, Nichols DA, Shaw EG: Interim report on the radiosurgical treatment of cerebral arteriovenous malformations. The influence of size, dose, time, and technical factors on obliteration rate. J Neurosurg 83:832-837, 1995

Manuscript submitted June 17, 2014.

Accepted July 24, 2014.

Please include this information when citing this paper: DOI: 10.3171/2014.7.GKS141297.

Address correspondence to: Hideyuki Kano, M.D., Ph.D., Department of Neurological Surgery, University of Pittsburgh, Ste. B-400, UPMC Presbyterian, 200 Lothrop St., Pittsburgh, PA 15213. email: kanoh@upmc.edu. 